\title{
On bicomplex generalized Tetranacci quaternions
}

\author{
Yüksel Soykan ${ }^{1}$ and Erkan Taşdemir ${ }^{2}$ \\ ${ }^{1}$ Department of Mathematics, Art and Science Faculty \\ Zonguldak Bülent Ecevit University, 67100, Zonguldak, Turkey \\ e-mail: yuksel_soykan@hotmail.com \\ 2 Pınarhisar Vocational School of Higher Education \\ Kırklareli University, 39300, Kırklareli, Turkey \\ e-mail: erkantasdemirehotmail.com
}

Received: 28 November 2019

Revised: 10 July 2020

Accepted: 14 July 2020

\begin{abstract}
In this paper, we introduce the bicomplex generalized Tetranacci quaternions. Furthermore, we present some properties of these quaternions and derive relationships between them.
\end{abstract}

Keywords: Bicomplex Tetranacci numbers, Quaternions, Bicomplex Tetranacci quaternions, Bicomplex Tetranacci-Lucas quaternions.

2010 Mathematics Subject Classification: 11B39, 11B83, 17A45, 05A15.

\section{Introduction}

In this paper, we define bicomplex generalized Tetranacci quaternions by combining bicomplex numbers and generalized Tetranacci numbers and give some properties of them. Before giving their definition, we present some information on generalized Tetranacci numbers, and also on bicomplex numbers.

A generalized Tetranacci sequence $\left\{V_{n}\right\}_{n \geq 0}=\left\{V_{n}\left(V_{0}, V_{1}, V_{2}, V_{3}\right)\right\}_{n \geq 0}$ is defined by the fourth-order recurrence relations

$$
V_{n}=V_{n-1}+V_{n-2}+V_{n-3}+V_{n-4}
$$

with the initial values $V_{0}=c_{0}, V_{1}=c_{1}, V_{2}=c_{2}, V_{3}=c_{3}$ not all being zero.

This sequence has been studied by many authors and more detail can be found in the extensive literature dedicated to these sequences, see for example [10,13, 14, 18, 21,22]. 
The sequence $\left\{V_{n}\right\}_{n \geq 0}$ can be extended to negative subscripts by defining

$$
V_{-n}=-V_{-(n-1)}-V_{-(n-2)}-V_{-(n-3)}+V_{-(n-4)}
$$

for $n=1,2,3, \ldots$ Therefore, recurrence (1) holds for all integer $n$.

The first few generalized Tetranacci numbers with positive subscript and negative subscript are given in the following Table 1:

\begin{tabular}{|c||c|c|}
\hline$n$ & $V_{n}$ & $V_{-n}$ \\
\hline \hline 0 & $c_{0}$ & $c_{0}$ \\
1 & $c_{1}$ & $c_{3}-c_{2}-c_{1}-c_{0}$ \\
2 & $c_{2}$ & $2 c_{2}-c_{3}$ \\
3 & $c_{3}$ & $2 c_{1}-c_{2}$ \\
4 & $c_{0}+c_{1}+c_{2}+c_{3}$ & $2 c_{0}-c_{1}$ \\
5 & $c_{0}+2 c_{1}+2 c_{2}+2 c_{3}$ & $2 c_{3}-2 c_{2}-2 c_{1}-3 c_{0}$ \\
6 & $2 c_{0}+3 c_{1}+4 c_{2}+4 c_{3}$ & $c_{0}+c_{1}+5 c_{2}-3 c_{3}$ \\
7 & $4 c_{0}+6 c_{1}+7 c_{2}+8 c_{3}$ & $4 c_{1}-4 c_{2}+c_{3}$ \\
8 & $8 c_{0}+12 c_{1}+14 c_{2}+15 c_{3}$ & $4 c_{0}-4 c_{1}+c_{2}$ \\
\hline
\end{tabular}

Table 1. A few generalized Tetranacci numbers

If we set $V_{0}=0, V_{1}=1, V_{2}=1, V_{3}=2$, then $\left\{V_{n}\right\}$ is the well-known Tetranacci sequence and if we set $V_{0}=4, V_{1}=1, V_{2}=3, V_{3}=7$ then $\left\{V_{n}\right\}$ is the well-known Tetranacci-Lucas sequence. In other words, Tetranacci sequence $\left\{M_{n}\right\}_{n \geq 0}$ and Tetranacci-Lucas sequence $\left\{R_{n}\right\}_{n \geq 0}$ are defined by the fourth-order recurrence relations

$$
M_{n}=M_{n-1}+M_{n-2}+M_{n-3}+M_{n-4}, \quad M_{0}=0, M_{1}=1, M_{2}=1, M_{3}=2
$$

and

$$
R_{n}=R_{n-1}+R_{n-2}+R_{n-3}+R_{n-4}, \quad R_{0}=4, R_{1}=1, R_{2}=3, R_{3}=7 .
$$

The sequences $\left\{M_{n}\right\}_{n \geq 0}$ and $\left\{R_{n}\right\}_{n \geq 0}$ can be extended to negative subscripts by defining

$$
M_{-n}=-M_{-(n-1)}-M_{-(n-2)}-M_{-(n-3)}+M_{-(n-4)}
$$

and

$$
R_{-n}=-R_{-(n-1)}-R_{-(n-2)}-R_{-(n-3)}+R_{-(n-4)}
$$

for $n=1,2,3, \ldots$ respectively. Therefore, recurrences (2) and (3) hold for all integer $n$.

It is well known that for all integers $n$, usual Tetranaci and Tetranacci-Lucas numbers can be expressed using Binet's formulas

$$
\begin{aligned}
M_{n}= & \frac{\alpha^{n+2}}{(\alpha-\beta)(\alpha-\gamma)(\alpha-\delta)}+\frac{\beta^{n+2}}{(\beta-\alpha)(\beta-\gamma)(\beta-\delta)} \\
& +\frac{\gamma^{n+2}}{(\gamma-\alpha)(\gamma-\beta)(\gamma-\delta)}+\frac{\delta^{n+2}}{(\delta-\alpha)(\delta-\beta)(\delta-\gamma)}
\end{aligned}
$$

(see for example [10] or [23]) 
or

$$
M_{n}=\frac{\alpha-1}{5 \alpha-8} \alpha^{n-1}+\frac{\beta-1}{5 \beta-8} \beta^{n-1}+\frac{\gamma-1}{5 \gamma-8} \gamma^{n-1}+\frac{\delta-1}{5 \delta-8} \delta^{n-1}
$$

(see for example [6])

and

$$
R_{n}=\alpha^{n}+\beta^{n}+\gamma^{n}+\delta^{n}
$$

respectively, where $\alpha, \beta, \gamma$ and $\delta$ are the roots of the equation $x^{4}-x^{3}-x^{2}-x-1=0$. Moreover,

$$
\begin{aligned}
\alpha & =\frac{1}{4}+\frac{1}{2} \omega+\frac{1}{2} \sqrt{\frac{11}{4}-\omega^{2}+\frac{13}{4} \omega^{-1}}, \\
\beta & =\frac{1}{4}+\frac{1}{2} \omega-\frac{1}{2} \sqrt{\frac{11}{4}-\omega^{2}+\frac{13}{4} \omega^{-1}}, \\
\gamma & =\frac{1}{4}-\frac{1}{2} \omega+\frac{1}{2} \sqrt{\frac{11}{4}-\omega^{2}-\frac{13}{4} \omega^{-1}}, \\
\delta & =\frac{1}{4}-\frac{1}{2} \omega-\frac{1}{2} \sqrt{\frac{11}{4}-\omega^{2}-\frac{13}{4} \omega^{-1}},
\end{aligned}
$$

where

$$
\omega=\sqrt{\frac{11}{12}+\left(\frac{-65}{54}+\sqrt{\frac{563}{108}}\right)^{1 / 3}+\left(\frac{-65}{54}-\sqrt{\frac{563}{108}}\right)^{1 / 3}} .
$$

We present Binet's formula of the generalized Tetranacci sequence.

Corollary 1.1. The Binet's formula of the generalized Tetranacci sequence $\left\{V_{n}\right\}$ is given as

$$
V_{n}=A \alpha^{n-6}+B \beta^{n-6}+C \gamma^{n-6}+D \delta^{n-6}
$$

where

$$
\begin{aligned}
A & =\frac{\alpha-1}{5 \alpha-8}\left(V_{3} \alpha^{3}+\left(V_{0}+V_{1}+V_{2}\right) \alpha^{2}+\left(V_{1}+V_{2}\right) \alpha+V_{2}\right), \\
B & =\frac{\beta-1}{5 \beta-8}\left(V_{3} \beta^{3}+\left(V_{0}+V_{1}+V_{2}\right) \beta^{2}+\left(V_{1}+V_{2}\right) \beta+V_{2}\right), \\
C & =\frac{\gamma-1}{5 \gamma-8}\left(V_{3} \gamma^{3}+\left(V_{0}+V_{1}+V_{2}\right) \gamma^{2}+\left(V_{1}+V_{2}\right) \gamma+V_{2}\right), \\
D & =\frac{\delta-1}{5 \delta-8}\left(V_{3} \delta^{3}+\left(V_{0}+V_{1}+V_{2}\right) \delta^{2}+\left(V_{1}+V_{2}\right) \delta+V_{2}\right) .
\end{aligned}
$$

Proof. For a proof see [19, Corollary 1.3.].

In fact, Corollary 1.1 is a special case of a result in [3, Remark 2.3.] .

Note that the Binet form of a sequence satisfying (1) for non-negative integers is valid for all integers $n$, for a proof of this result see [11]. This result of Howard and Saidak [11] is even true in the case of higher-order recurrence relations.

Next, we give the ordinary generating function $\sum_{n=0}^{\infty} a_{n} x^{n}$ of the sequence $V_{n}$.

Lemma 1.2. Suppose that $f_{V_{n}}(x)=\sum_{n=0}^{\infty} a_{n} x^{n}$ is the ordinary generating function of the generalized Tetranacci sequence $\left\{V_{n}\right\}_{n \geq 0}$. Then $f_{V_{n}}(x)$ is given by

$$
f_{V_{n}}(x)=\frac{V_{0}+\left(V_{1}-V_{0}\right) x+\left(V_{2}-V_{1}-V_{0}\right) x^{2}+\left(V_{3}-V_{2}-V_{1}-V_{0}\right) x^{3}}{1-x-x^{2}-x^{3}-x^{4}} .
$$


Proof. Using (1) and some calculation, we obtain

$$
\begin{aligned}
f_{V_{n}}(x)-x f_{V_{n}}(x)-x^{2} f_{V_{n}}(x)-x^{3} f_{V_{n}}(x)-x^{4} f_{V_{n}}(x)= & V_{0}+\left(V_{1}-V_{0}\right) x \\
& +\left(V_{2}-V_{1}-V_{0}\right) x^{2} \\
& +\left(V_{3}-V_{2}-V_{1}-V_{0}\right) x^{3}
\end{aligned}
$$

which gives (5).

The previous Lemma gives the following results as particular examples: generating function of the Tetranacci sequence $M_{n}$ is

$$
f_{M_{n}}(x)=\sum_{n=0}^{\infty} M_{n} x^{n}=\frac{x}{1-x-x^{2}-x^{3}-x^{4}}
$$

and generating function of the Tetranacci-Lucas sequence $R_{n}$ is

$$
f_{R_{n}}(x)=\sum_{n=0}^{\infty} R_{n} x^{n}=\frac{4-3 x-2 x^{2}-x^{3}}{1-x-x^{2}-x^{3}-x^{4}} .
$$

The bicomplex numbers (quaternions) are defined by the four bases $1, i, j, i j$ where $i, j$ and ij satisfy the following properties:

$$
i^{2}=-1, j^{2}=-1, i j=j i .
$$

A bicomplex number can be expressed as follows:

$$
q=a_{0}+i a_{1}+j a_{2}+i j a_{3}=\left(a_{0}+i a_{1}\right)+j\left(a_{2}+i a_{3}\right)=z_{0}+j z_{1}
$$

where $a_{0}, a_{1}, a_{2}, a_{3}$ are real numbers and $z_{0}, z_{1}$ are complex numbers. So the set of bicomplex number is

$$
\mathbb{B C}=\left\{z_{0}+j z_{1}: z_{0}, z_{1} \in \mathbb{C}, j^{2}=-1\right\} .
$$

Moreover, for any bicomplex numbers

$$
q=a_{0}+i a_{1}+j a_{2}+i j a_{3}
$$

and

$$
p=b_{0}+i b_{1}+j b_{2}+i j b_{3}
$$

and scalar $\lambda \in \mathbb{R}$, the addition, subtraction and multiplication with scalar are defined as componentwise, i.e

$$
\begin{aligned}
q+p & =\left(a_{0}+b_{0}\right)+i\left(a_{1}+b_{1}\right)+j\left(a_{2}+b_{2}\right)+i j\left(a_{3}+b_{3}\right), \\
q-p & =\left(a_{0}-b_{0}\right)+i\left(a_{1}-b_{1}\right)+j\left(a_{2}-b_{2}\right)+i j\left(a_{3}-b_{3}\right), \\
\lambda q & =\lambda a_{0}+i \lambda a_{1}+j \lambda a_{2}+i j \lambda a_{3}
\end{aligned}
$$

respectively, and product (multiplication) is defined as follows:

$$
\begin{aligned}
q \times p= & \left(a_{0} b_{0}-a_{1} b_{1}-a_{2} b_{2}+a_{3} b_{3}\right)+i\left(a_{0} b_{1}+a_{1} b_{0}-a_{2} b_{3}-a_{3} b_{2}\right) \\
& +j\left(a_{0} b_{2}-a_{1} b_{3}+a_{2} b_{0}-a_{3} b_{1}\right)+i j\left(a_{0} b_{3}+a_{1} b_{2}+a_{2} b_{1}+a_{3} b_{0}\right) .
\end{aligned}
$$


There are three different conjugations (involutions) for bicomplex numbers, namely

$$
\begin{aligned}
q_{i}^{*} & =a_{0}-i a_{1}+j a_{2}-i j a_{3}=\overline{z_{0}}+j \overline{z_{1}}, \\
q_{j}^{*} & =a_{0}+i a_{1}-j a_{2}-i j a_{3}=z_{0}-j z_{1}, \\
q_{i j}^{*} & =a_{0}-i a_{1}-j a_{2}+i j a_{3}=\overline{z_{0}}-j \overline{z_{1}},
\end{aligned}
$$

for $q=a_{0}+i a_{1}+j a_{2}+i j a_{3}$. The squares of norms of the bicomplex numbers which arise from the definitions of conjugations are given by

$$
\begin{aligned}
& N_{i}^{2}(q)=\left|q_{i} \times q_{i}^{*}\right|:=\left|a_{0}^{2}+a_{1}^{2}-a_{2}^{2}-a_{3}^{2}+2 j\left(a_{0} a_{2}+a_{1} a_{3}\right)\right|, \\
& N_{j}^{2}(q)=\left|q_{j} \times q_{j}^{*}\right|:=\left|a_{0}^{2}+a_{1}^{2}-a_{2}^{2}-a_{3}^{2}+2 i\left(a_{0} a_{1}+a_{2} a_{3}\right)\right|, \\
& N_{i j}^{2}(q)=\left|q_{i j} \times q_{i j}^{*}\right|:=\left|a_{0}^{2}+a_{1}^{2}+a_{2}^{2}+a_{3}^{2}+2 i j\left(a_{0} a_{3}-a_{2} a_{1}\right)\right| .
\end{aligned}
$$

For more details about these type of numbers (quaternions), we refer to, for example, the works [7, 17], among others.

\section{The bicomplex generalized Tetranacci and Tetranacci-Lucas quaternions and their generating functions, Binet's formulas and summations formulas}

In this section we define the bicomplex generalized Tetranacci quaternions and give generating functions and Binet formulas for them. First, we give some information about bicomplex type quaternion sequences from the literature.

Nurkan and Güven [15] (see also [16]) introduced $n$-th bicomplex Fibonacci and $n$-th bicomplex Lucas numbers (quaternions) as

$$
B F_{n}=F_{n}+F_{n+1} i+F_{n+2} j+F_{n+3} i j
$$

and

$$
B L_{n}=L_{n}+L_{n+1} i+L_{n+2} j+L_{n+3} i j
$$

respectively, where $F_{n}$ and $L_{n}$ are the $n$-th Fibonacci and Lucas numbers, respectively. Various families of bicomplex number (quaternion) sequences have been defined and studied by a number of authors. See, for example, [1,2,4,8,9] for second order bicomplex quaternion sequences and $[5,12]$ for third order bicomplex quaternion sequences.

Soykan [20] introduced the bicomplex Tetranacci and Tetranacci-Lucas quaternions as fourth order bicomplex quaternion sequences.

We now define bicomplex generalized Tetranacci quaternions over the algebra $\mathbb{B C}$.

Definition 2.1. The $n$-th bicomplex generalized Tetranacci quaternion is

$$
\mathbb{B} \mathbb{C} V_{n}=V_{n}+i V_{n+1}+j V_{n+2}+i j V_{n+3}
$$

As special cases, the $n$-th Tetranacci quaternion and the $n$-th Tetranacci-Lucas quaternion are given as

$$
\mathbb{B C} M_{n}=M_{n}+i M_{n+1}+j M_{n+2}+i j M_{n+3}
$$


and

$$
\mathbb{B C} R_{n}=R_{n}+i R_{n+1}+j R_{n+2}+i j R_{n+3},
$$

respectively. It can be easily shown that $\left\{\mathbb{B C C} V_{n}\right\}_{n \geq 0}$ can also be defined by the recurrence relations:

$$
\mathbb{B} \mathbb{C} V_{n}=\mathbb{B} \mathbb{C} V_{n-1}+\mathbb{B} \mathbb{C} V_{n-2}+\mathbb{B} \mathbb{C} V_{n-3}+\mathbb{B} \mathbb{C} V_{n-4}
$$

with the intial conditions $\mathbb{B} \mathbb{C} V_{0}, \mathbb{B} \mathbb{C} V_{1}, \mathbb{B C} V_{2}, \mathbb{B C} V_{3}$ (see Table 1).

The sequence $\left\{\mathbb{B} \mathbb{C} V_{n}\right\}_{n \geq 0}$ can be extended to negative subscripts by defining

$$
\mathbb{B} \mathbb{C} V_{-n}=-\mathbb{B C} V_{-(n-1)}-\mathbb{B} \mathbb{C} V_{-(n-2)}-\mathbb{B} \mathbb{C} V_{-(n-3)}+\mathbb{B} \mathbb{C} V_{-(n-4)}
$$

for $n=1,2,3, \ldots$ respectively. Therefore, recurrence (7) holds for all integer $n$.

The first few bicomplex generalized Tetranacci quaternions with positive subscript and negative subscript are given in the following Table 2:

\begin{tabular}{|c|c|}
\hline$n$ & $\mathbb{B} \mathbb{C} V_{n}$ \\
\hline \hline-5 & $\left(2 c_{3}-2 c_{2}-2 c_{1}-3 c_{0}\right)+i\left(2 c_{0}-c_{1}\right)+j\left(2 c_{1}-c_{2}\right)+i j\left(2 c_{2}-c_{3}\right)$ \\
-4 & $\left(2 c_{0}-c_{1}\right)+i\left(2 c_{1}-c_{2}\right)+j\left(2 c_{2}-c_{3}\right)+i j\left(c_{3}-c_{2}-c_{1}-c_{0}\right)$ \\
-3 & $\left(2 c_{1}-c_{2}\right)+i\left(2 c_{2}-c_{3}\right)+j\left(c_{3}-c_{2}-c_{1}-c_{0}\right)+i j c_{0}$ \\
-2 & $\left(2 c_{2}-c_{3}\right)+i\left(c_{3}-c_{2}-c_{1}-c_{0}\right)+j c_{0}+i j c_{1}$ \\
-1 & $\left(c_{3}-c_{2}-c_{1}-c_{0}\right)+i c_{0}+j c_{1}+i j c_{2}$ \\
0 & $c_{0}+i c_{1}+j c_{2}+i j c_{3}$ \\
1 & $c_{1}+i c_{2}+j c_{3}+i j\left(c_{0}+c_{1}+c_{2}+c_{3}\right)$ \\
2 & $c_{2}+i c_{3}+j\left(c_{0}+c_{1}+c_{2}+c_{3}\right)+i j\left(c_{0}+c_{1}+c_{2}+c_{3}\right)$ \\
3 & $c_{3}+i\left(c_{0}+c_{1}+c_{2}+c_{3}\right)+j\left(c_{0}+2 c_{1}+2 c_{2}+2 c_{3}\right)+i j\left(2 c_{0}+3 c_{1}+4 c_{2}+4 c_{3}\right)$ \\
4 & $\left(c_{0}+c_{1}+c_{2}+c_{3}\right)+i\left(c_{0}+2 c_{1}+2 c_{2}+2 c_{3}\right)+j\left(2 c_{0}+3 c_{1}+4 c_{2}+4 c_{3}\right)$ \\
& $+i j\left(4 c_{0}+6 c_{1}+7 c_{2}+8 c_{3}\right)$ \\
\hline
\end{tabular}

Table 2. Bicomplex generalized Tetranacci quaternions

For two bicomplex generalized Tetranacci quaternions $\mathbb{B} \mathbb{C} V_{n}$ and $\mathbb{B} \mathbb{C} V_{k}$ and for scalar $\lambda \in \mathbb{R}$, the addition, subtraction and multiplication with scalar are defined as componentwise, i.e.,

$$
\begin{aligned}
\mathbb{B} C V_{n}+\mathbb{B} \mathbb{C} V_{k} & =\left(V_{n}+V_{k}\right)+i\left(V_{n+1}+V_{k+1}\right)+j\left(V_{n+2}+V_{k+2}\right)+i j\left(V_{n+3}+V_{k+3}\right), \\
\mathbb{B C C} V_{n}-\mathbb{B} \mathbb{C} V_{k} & =\left(V_{n}-V_{k}\right)+i\left(V_{n+1}-V_{k+1}\right)+j\left(V_{n+2}-V_{k+2}\right)+i j\left(V_{n+3}-V_{k+3}\right), \\
\lambda \mathbb{B} \mathbb{C} V_{n} & =\lambda V_{n}+i \lambda V_{n+1}+j \lambda V_{n+2}+i j \lambda V_{n+3}
\end{aligned}
$$

respectively, and product (multiplication) is defined as follows:

$$
\begin{aligned}
\mathbb{B C} V_{n} \times \mathbb{B} \mathbb{C} V_{k}= & \left(V_{n} V_{k}-V_{n+1} V_{k+1}-V_{n+2} V_{k+2}+V_{n+3} V_{k+3}\right) \\
& +i\left(V_{n} V_{k+1}+V_{n+1} V_{k}-V_{n+2} V_{k+3}-V_{n+3} V_{k+2}\right) \\
& +j\left(V_{n} V_{k+2}-V_{n+1} V_{k+3}+V_{n+2} V_{k}-V_{n+3} V_{k+1}\right) \\
& +i j\left(V_{n} V_{k+3}+V_{n+1} V_{k+2}+V_{n+2} V_{k+1}+V_{n+3} V_{k}\right) \\
= & \mathbb{B} \mathbb{C} V_{k} \times \mathbb{B} \mathbb{C} V_{n} .
\end{aligned}
$$


Moreover, three different conjugations for the bicomplex Tribonacci quaternion $\mathbb{B C} V_{n}=V_{n}+i V_{n+1}+j V_{n+2}+i j V_{n+3}$ are given as

$$
\begin{aligned}
\left(\mathbb{B C} V_{n}\right)_{i}^{*} & =V_{n}-i V_{n+1}+j V_{n+2}-i j V_{n+3}, \\
\left(\mathbb{B C C} V_{n}\right)_{j}^{*} & =V_{n}+i V_{n+1}-j V_{n+2}-i j V_{n+3}, \\
\left(\mathbb{B C C} V_{n}\right)_{i j}^{*} & =V_{n}-i V_{n+1}-j V_{n+2}+i j V_{n+3},
\end{aligned}
$$

and the squares of norms of the bicomplex Tribonacci quaternion are given by

$$
\begin{aligned}
N_{i}^{2}\left(\mathbb{B C} V_{n}\right) & =\left|\left(\mathbb{B C} V_{n}\right)_{i} \times\left(\mathbb{B C C} V_{n}\right)_{i}^{*}\right| \\
& :=\left|V_{n}^{2}+V_{n+1}^{2}-V_{n+2}^{2}-V_{n+3}^{2}+2 j\left(V_{n} V_{n+2}+V_{n+1} V_{n+3}\right)\right|, \\
N_{j}^{2}\left(\mathbb{B C C} V_{n}\right)= & \left|\left(\mathbb{B} \mathbb{C} V_{n}\right)_{j} \times\left(\mathbb{B C} V_{n}\right)_{j}^{*}\right| \\
: & =\left|V_{n}^{2}+V_{n+1}^{2}-V_{n+2}^{2}-V_{n+3}^{2}+2 i\left(V_{n} V_{n+1}+V_{n+2} V_{n+3}\right)\right|, \\
N_{i j}^{2}\left(\mathbb{B C C} V_{n}\right)= & \left|\left(\mathbb{B} \mathbb{C} V_{n}\right)_{i j} \times\left(\mathbb{B} \mathbb{C} V_{n}\right)_{i j}^{*}\right| \\
: & =\left|V_{n}^{2}+V_{n+1}^{2}+V_{n+2}^{2}+V_{n+3}^{2}+2 i j\left(V_{n} V_{n+3}-V_{n+2} V_{n+1}\right)\right| .
\end{aligned}
$$

Now, we will state Binet's formula for the bicomplex generalized Tetranacci quaternions and in the rest of the paper we fix the following notations.

$$
\begin{aligned}
& \widehat{\alpha}=1+i \alpha+j \alpha^{2}+i j \alpha^{3}, \\
& \widehat{\beta}=1+i \beta+j \beta^{2}+i j \beta^{3}, \\
& \widehat{\gamma}=1+i \gamma+j \gamma^{2}+i j \gamma^{3} . \\
& \widehat{\delta}=1+i \delta+j \delta^{2}+i j \delta^{3} .
\end{aligned}
$$

Theorem 2.1 (Binet's Formula). For any integer $n$, the $n$-th bicomplex generalized Tetranacci quaternion is

$$
\mathbb{B} \mathbb{C} V_{n}=A \widehat{\alpha} \alpha^{n-6}+B \widehat{\beta} \beta^{n-6}+C \widehat{\gamma} \gamma^{n-6}+D \widehat{\delta} \delta^{n-6}
$$

where $A, B, C$ and $D$ are as in Corollary 1.1.

Proof. Using Binet's formula of the generalized Tetranacci numbers, we obtain

$$
\begin{aligned}
\mathbb{B C C} V_{n}= & V_{n}+i V_{n+1}+j V_{n+2}+i j V_{n+3} \\
= & A \alpha^{n-6}+B \beta^{n-6}+C \gamma^{n-6}+D \delta^{n-6}+i\left(A \alpha^{n-5}+B \beta^{n-5}+C \gamma^{n-5}+D \delta^{n-5}\right) \\
& +j\left(A \alpha^{n-4}+B \beta^{n-4}+C \gamma^{n-4}+D \delta^{n-4}\right)+i j\left(A \alpha^{n-3}+B \beta^{n-3}+C \gamma^{n-3}+D \delta^{n-3}\right) \\
= & A \widehat{\alpha} \alpha^{n-6}+B \widehat{\beta} \beta^{n-6}+C \widehat{\gamma} \gamma^{n-6}+D \widehat{\delta} \delta^{n-6} .
\end{aligned}
$$

This proves (8).

As special cases, for any integer $n$, the Binet's Formula of $n$-th bicomplex Tetranacci quaternion is

$$
\mathbb{B} \mathbb{C} M_{n}=\frac{\alpha-1}{5 \alpha-8} \widehat{\alpha} \alpha^{n-1}+\frac{\beta-1}{5 \beta-8} \widehat{\beta} \beta^{n-1}+\frac{\gamma-1}{5 \gamma-8} \widehat{\gamma} \gamma^{n-1}+\frac{\delta-1}{5 \delta-8} \widehat{\delta} \delta^{n-1}
$$

and the Binet's Formula of $n$-th bicomplex Tetranacci-Lucas quaternion is

$$
\mathbb{B} \mathbb{C} R_{n}=\widehat{\alpha} \alpha^{n}+\widehat{\beta} \beta^{n}+\widehat{\gamma} \gamma^{n}+\widehat{\delta} \delta^{n} .
$$


Next, we present generating functions.

Theorem 2.2. The generating function for the bicomplex generalized Tetranacci quaternions is

$$
\sum_{n=0}^{\infty} \widehat{V}_{n} x^{n}=\frac{\mathbb{B} \mathbb{C} V_{0}+\left(\mathbb{B} \mathbb{C} V_{1}-\mathbb{B} \mathbb{C} V_{0}\right) x+\left(\mathbb{B} \mathbb{C} V_{2}-\mathbb{B} \mathbb{C} V_{1}-\mathbb{B} \mathbb{C} V_{0}\right) x^{2}+\mathbb{B} \mathbb{C} V_{-1} x^{3}}{1-x-x^{2}-x^{3}-x^{4}}
$$

Proof. Let

$$
g(x)=\sum_{n=0}^{\infty} \mathbb{B} \mathbb{C} V_{n} x^{n}
$$

be the generating function of the bicomplex Tetranacci quaternions. Then using the definition of the bicomplex Tetranacci quaternions, and substracting $x g(x), x^{2} g(x), x^{3} g(x)$ and $x^{4} g(x)$ from $g(x)$ and using the recurrence relation $\mathbb{B} \mathbb{C} V_{n}=\mathbb{B C C} V_{n-1}+\mathbb{B C C} V_{n-2}+\mathbb{B} \mathbb{C} V_{n-3}+\mathbb{B} \mathbb{C} V_{n-4}$, we obtain

$$
\begin{aligned}
\left(1-x-x^{2}-x^{3}-x^{4}\right) g(x)= & \mathbb{B C} V_{0}+\left(\mathbb{B C} V_{1}-\mathbb{B} \mathbb{C} V_{0}\right) x+\left(\mathbb{B C} V_{2}-\mathbb{B C} V_{1}-\mathbb{B C} V_{0}\right) x^{2} \\
& +\left(\mathbb{B C} V_{3}-\mathbb{B C} V_{2}-\mathbb{B} \mathbb{C} V_{1}-\mathbb{B} \mathbb{C} V_{0}\right) x^{3}
\end{aligned}
$$

Note that we used the recurrence relation $\mathbb{B C} V_{n}=\mathbb{B C} V_{n-1}+\mathbb{B C} V_{n-2}+\mathbb{B C} V_{n-3}+\mathbb{B} \mathbb{C} V_{n-4}$. Rearranging above equation and using $\mathbb{B} \mathbb{C} V_{3}=\mathbb{B C} V_{2}+\mathbb{B C C} V_{1}+\mathbb{B C C} V_{0}+\mathbb{B C} V_{-1}$, we get

$$
g(x)=\frac{\mathbb{B} \mathbb{C} V_{0}+\left(\mathbb{B} \mathbb{C} V_{1}-\mathbb{B} \mathbb{C} V_{0}\right) x+\left(\mathbb{B} \mathbb{C} V_{2}-\mathbb{B} \mathbb{C} V_{1}-\mathbb{B} \mathbb{C} V_{0}\right) x^{2}+\mathbb{B} \mathbb{C} V_{-1} x^{3}}{1-x-x^{2}-x^{3}-x^{4}}
$$

As special cases, the generating functions for the bicomplex Tetranacci and Tetranacci-Lucas quaternions, respectively, are

$$
\sum_{n=0}^{\infty} \mathbb{B} \mathbb{C} M_{n} x^{n}=\frac{(i+j+2 i j)+(1+j+2 i j) x+(j+2 i j) x^{2}+(j+i j) x^{3}}{1-x-x^{2}-x^{3}-x^{4}}
$$

and

$$
\sum_{n=0}^{\infty} \mathbb{B} \mathbb{C} R_{n} x^{n}=\frac{c_{1}}{1-x-x^{2}-x^{3}-x^{4}}
$$

where

$c_{1}=(4+i+3 j+7 i j)+(-3+2 i+4 j+8 i j) x+(-2+3 i+5 j+4 i j) x^{2}+(-1+4 i+j+3 i j) x^{3}$.

Next we present some summation formulas of Tetranacci numbers.

Lemma 2.3. For $n \geq 1$ we have the following formulas:

(a) $\sum_{p=1}^{n} V_{p}=\frac{1}{3}\left(V_{n+2}+2 V_{n}+V_{n-1}-V_{0}+V_{1}-V_{3}\right)$

(b) $\sum_{p=1}^{n} V_{2 p+1}=\frac{1}{3}\left(2 V_{2 n+2}+V_{2 n}-V_{2 n-1}-2 V_{0}-V_{1}-3 V_{2}+V_{3}\right)$

(c) $\sum_{p=1}^{n} V_{2 p}=\frac{1}{3}\left(2 V_{2 n+1}+V_{2 n-1}-V_{2 n-2}+V_{0}-V_{1}+3 V_{2}-2 V_{3}\right)$. 
The above Lemma is given in Soykan [19, Theorem 2.6] .

Note that from above Lemma we have

$$
\begin{aligned}
\sum_{p=0}^{n} V_{p} & =V_{0}+\sum_{p=1}^{n} V_{p}=V_{0}+\frac{1}{3}\left(V_{n+2}+2 V_{n}+V_{n-1}-V_{0}+V_{1}-V_{3}\right) \\
& =\frac{1}{3}\left(V_{n+2}+2 V_{n}+V_{n-1}+2 V_{0}+V_{1}-V_{3}\right)
\end{aligned}
$$

and

$$
\begin{aligned}
\sum_{p=0}^{n} V_{2 p+1} & =V_{1}+\sum_{p=1}^{n} V_{2 p+1} \\
& =V_{1}+\frac{1}{3}\left(2 V_{2 n+2}+V_{2 n}-V_{2 n-1}-2 V_{0}-V_{1}-3 V_{2}+V_{3}\right) \\
& =\frac{1}{3}\left(2 V_{2 n+2}+V_{2 n}-V_{2 n-1}-2 V_{0}+2 V_{1}-3 V_{2}+V_{3}\right)
\end{aligned}
$$

and

$$
\begin{aligned}
\sum_{p=0}^{n} V_{2 p} & =V_{0}+\sum_{p=1}^{n} V_{2 p} \\
& =V_{0}+\frac{1}{3}\left(2 V_{2 n+1}+V_{2 n-1}-V_{2 n-2}+V_{0}-V_{1}+3 V_{2}-2 V_{3}\right) \\
& =\frac{1}{3}\left(2 V_{2 n+1}+V_{2 n-1}-V_{2 n-2}+4 V_{0}-V_{1}+3 V_{2}-2 V_{3}\right) .
\end{aligned}
$$

In the following Theorem, we give some summation formulas of bicomplex generalized Tetranacci quaternions.

Theorem 2.4. For $n \geq 0$ we have the following formulas:

(a)

$$
\sum_{p=0}^{n} \mathbb{B} \mathbb{C} V_{p}=\frac{1}{3}\left(\mathbb{B} \mathbb{C} V_{n+2}+2 \mathbb{B} \mathbb{C} V_{n}+\mathbb{B} \mathbb{C} V_{n-1}+c\right)
$$

where

$$
\begin{aligned}
c= & 2 V_{0}+V_{1}-V_{3}+i\left(-V_{0}+V_{1}-V_{3}\right)+j\left(-V_{0}-2 V_{1}-V_{3}\right) \\
& +i j\left(-V_{0}-2 V_{1}-3 V_{2}-V_{3}\right) .
\end{aligned}
$$

(b)

$$
\sum_{p=0}^{n} \mathbb{B} \mathbb{C} V_{2 p+1}=\frac{1}{3}\left(2 \mathbb{B} \mathbb{C} V_{2 n+2}+\mathbb{B} \mathbb{C} V_{2 n}-\mathbb{B} \mathbb{C} V_{2 n-1}+d\right)
$$

where

$$
\begin{aligned}
d= & \left(-2 V_{0}+2 V_{1}-3 V_{2}+V_{3}\right)+i\left(V_{0}-V_{1}+3 V_{2}-2 V_{3}\right) \\
& +j\left(-2 V_{0}-V_{1}-3 V_{2}+V_{3}\right)+i j\left(V_{0}-V_{1}-2 V_{3}\right)
\end{aligned}
$$


(c)

$$
\sum_{p=0}^{n} \mathbb{B} \mathbb{C} V_{2 p}=\frac{1}{3}\left(2 \mathbb{B} \mathbb{C} V_{2 n+1}+\mathbb{B} \mathbb{C} V_{2 n-1}-\mathbb{B} \mathbb{C} V_{2 n-2}+e\right)
$$

where

$$
\begin{aligned}
e= & \left(4 V_{0}-V_{1}+3 V_{2}-2 V_{3}\right)+\left(-2 V_{0}+2 V_{1}-3 V_{2}+V_{3}\right) i \\
& +\left(V_{0}-V_{1}+3 V_{2}-2 V_{3}\right) j+\left(-2 V_{0}-V_{1}-3 V_{2}+V_{3}\right) i j
\end{aligned}
$$

Proof. (a) Using (6), we obtain

$$
\begin{aligned}
\sum_{p=0}^{n} \mathbb{B} \mathbb{C} V_{p}= & \sum_{p=0}^{n} V_{p}+i \sum_{p=0}^{n} V_{p+1}+j \sum_{p=0}^{n} V_{p+2}+i j \sum_{p=0}^{n} V_{p+3} \\
= & \left(V_{0}+\ldots+V_{n}\right)+i\left(V_{1}+\ldots+V_{n+1}\right) \\
& +j\left(V_{2}+\ldots+V_{n+2}\right)+i j\left(V_{3}+\ldots+V_{n+3}\right) .
\end{aligned}
$$

and so

$$
\begin{aligned}
3 \sum_{p=0}^{n} \mathbb{B} \mathbb{C} V_{p}= & \left(V_{n+2}+2 V_{n}+V_{n-1}+2 V_{0}+V_{1}-V_{3}\right) \\
& +i\left(V_{n+3}+2 V_{n+1}+V_{n}+2 V_{0}+V_{1}-V_{3}-3 V_{0}\right) \\
& +j\left(V_{n+4}+2 V_{n+2}+V_{n+1}+2 V_{0}+V_{1}-V_{3}-3\left(V_{0}+V_{1}\right)\right) \\
& +k\left(V_{n+5}+2 V_{n+3}+V_{n+2}+2 V_{0}+V_{1}-V_{3}-3\left(V_{0}+V_{1}+V_{2}\right)\right) \\
= & \mathbb{B} \mathbb{C} V_{n+2}+2 \mathbb{B} \mathbb{C} V_{n}+\mathbb{B} \mathbb{C} V_{n-1}+c
\end{aligned}
$$

where

$$
\begin{aligned}
c= & 2 V_{0}+V_{1}-V_{3}+i\left(2 V_{0}+V_{1}-V_{3}-3 V_{0}\right)+j\left(2 V_{0}+V_{1}-V_{3}-3\left(V_{0}+V_{1}\right)\right) \\
& +i j\left(2 V_{0}+V_{1}-V_{3}-3\left(V_{0}+V_{1}+V_{2}\right)\right) \\
= & 2 V_{0}+V_{1}-V_{3}+i\left(-V_{0}+V_{1}-V_{3}\right) \\
& +j\left(-V_{0}-2 V_{1}-V_{3}\right)+i j\left(-V_{0}-2 V_{1}-3 V_{2}-V_{3}\right)
\end{aligned}
$$

Hence

$$
\sum_{p=0}^{n} \mathbb{B} \mathbb{C} V_{p}=\frac{1}{3}\left(\mathbb{B} \mathbb{C} V_{n+2}+2 \mathbb{B} \mathbb{C} V_{n}+\mathbb{B} \mathbb{C} V_{n-1}+c\right)
$$

This proves (17).

(b) and (c) follows from the identities (15) and (16).

As special cases we have the following two corollaries.

Corollary 2.5. For $n \geq 0$ we have the following formulas:

(a)

$$
\sum_{p=0}^{n} \mathbb{B} \mathbb{C} M_{p}=\frac{1}{3}\left(\mathbb{B} \mathbb{C} M_{n+2}+2 \mathbb{B} \mathbb{C} M_{n}+\mathbb{B C} M_{n-1}-(1+i+4 j+7 i j)\right)
$$


(b)

$$
\sum_{p=0}^{n} \mathbb{B} \mathbb{C} M_{2 p+1}=\frac{1}{3}\left(2 \mathbb{B} \mathbb{C} M_{2 n+2}+\mathbb{B} \mathbb{C} M_{2 n}-\mathbb{B} \mathbb{C} M_{2 n-1}+(1-2 i-2 j-5 i j)\right) .
$$

(c)

$$
\sum_{p=0}^{n} \mathbb{B} \mathbb{C} M_{2 p}=\frac{1}{3}\left(2 \mathbb{B C} M_{2 n+1}+\mathbb{B} \mathbb{C} M_{2 n-1}-\mathbb{B C} M_{2 n-2}-(2-i+2 j+2 i j)\right) .
$$

Corollary 2.6. For $n \geq 0$ we have the following formulas:

(a)

$$
\sum_{p=0}^{n} \mathbb{B} \mathbb{C} R_{p}=\frac{1}{3}\left(\mathbb{B} \mathbb{C} R_{n+2}+2 \mathbb{B} \mathbb{C} R_{n}+\mathbb{B} \mathbb{C} R_{n-1}+(2-10 i-13 j-22 i j)\right) .
$$

(b)

$$
\sum_{p=0}^{n} \mathbb{B} \mathbb{C} R_{2 p+1}=\frac{1}{3}\left(2 \mathbb{B} \mathbb{C} R_{2 n+2}+\mathbb{B} \mathbb{C} R_{2 n}-\mathbb{B} \mathbb{C} R_{2 n-1}-(8+2 i+11 j+11 i j)\right) .
$$

(c)

$$
\sum_{p=0}^{n} \mathbb{B} \mathbb{C} R_{2 p}=\frac{1}{3}\left(2 \mathbb{B} \mathbb{C} R_{2 n+1}+\mathbb{B} \mathbb{C} R_{2 n-1}-\mathbb{B} \mathbb{C} R_{2 n-2}+(10-8 i-2 j-11 i j)\right) .
$$

\section{Five-diagonal matrix with fourth order sequences and applications}

In this section we give another way to obtain nth term of the bicomplex Tetranacci and TetranacciLucas quaternions. For this we need the following theorem.

Theorem 3.1. Let $\left\{x_{n}\right\}$ be any fourth-order linear sequence defined recursively as follows:

$$
x_{n}=r x_{n-1}+s x_{n-2}+t x_{n-3}+u x_{n-4}, \quad n \geq 4
$$

with the initial conditions $x_{0}=a, x_{1}=b, x_{2}=c, x_{3}=d$. Then for all $n \geq 0$, we have

$$
x_{n}=\left|\begin{array}{cccccccccccc}
a & -1 & 0 & 0 & 0 & 0 & 0 & \cdots & 0 & 0 & 0 & 0 \\
b & 0 & -1 & 0 & 0 & 0 & 0 & \cdots & 0 & 0 & 0 & 0 \\
c & 0 & 0 & -1 & 0 & 0 & 0 & \cdots & 0 & 0 & 0 & 0 \\
d & 0 & 0 & 0 & -1 & 0 & 0 & \cdots & 0 & 0 & 0 & 0 \\
0 & u & t & s & r & -1 & 0 & \cdots & 0 & 0 & 0 & 0 \\
0 & 0 & u & t & s & r & -1 & \cdots & 0 & 0 & 0 & 0 \\
\vdots & \vdots & \vdots & \vdots & \vdots & \vdots & \vdots & \ddots & \vdots & \vdots & \vdots & \vdots \\
0 & 0 & 0 & 0 & 0 & 0 & 0 & \cdots & s & r & -1 & 0 \\
0 & 0 & 0 & 0 & 0 & 0 & 0 & \cdots & t & s & r & -1 \\
0 & 0 & 0 & 0 & 0 & 0 & 0 & \cdots & u & t & s & r
\end{array}\right|_{(n+1) \times(n+1)}
$$


The proof of the above Theorem can be found in Soykan [20].

Note that in our cases $r=s=t=u=1$. As a corallary of the above theorem, in the following we present another way to obtain nth term of the bicomplex generalized Tetranacci quaternions.

Corollary 3.2. For all $n \geq 0$, we have

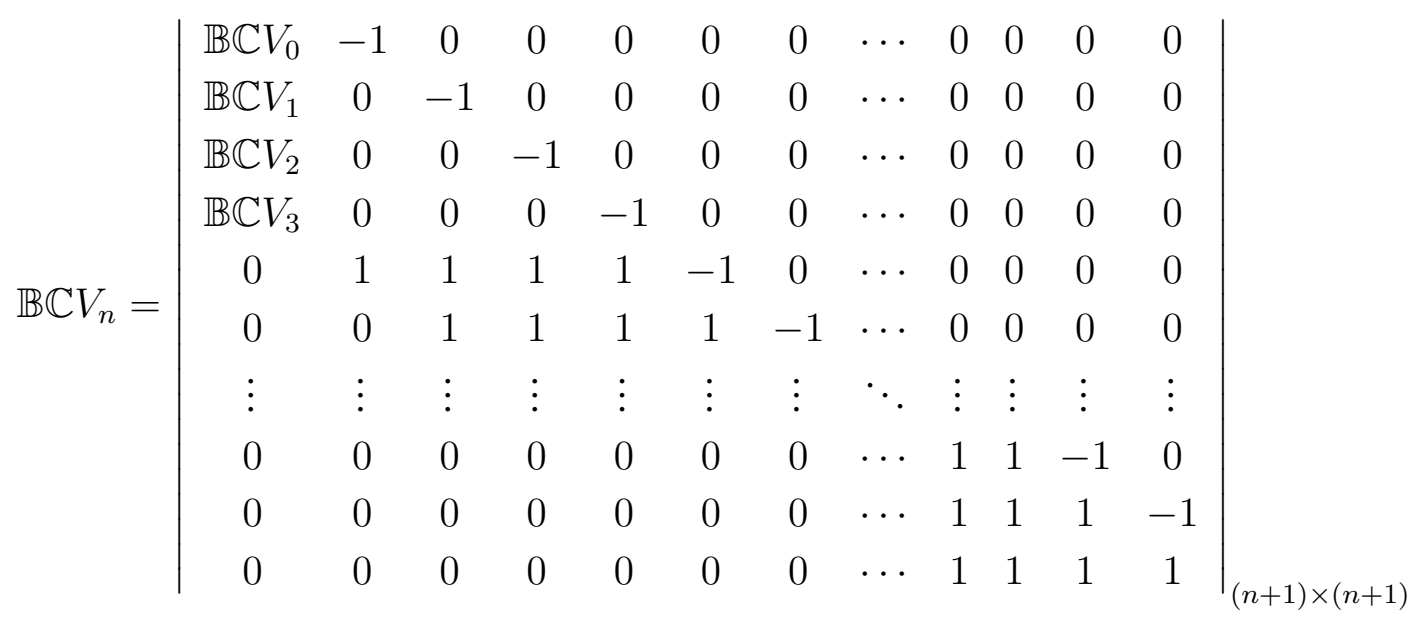

Proof. (a) follows from (7) and Theorem 3.1.

\section{References}

[1] Aydın, F. T. (2018). Bicomplex k-Fibonacci Quaternions, arXiv: 1810.05003 [math.NT].

[2] Aydın, F. T. (2018). On Bicomplex Pell and Pell-Lucas Numbers, Communications in Advanced Mathematical Sciences, 1 (2), 142-155.

[3] Bacani, J. B., \& Rabago, J. F. T. (2015). On Generalized Fibonacci Numbers, Applied Mathematical Sciences, 9 (25), 3611-3622.

[4] Catarino, P. (2019). Bicomplex k-Pell Quaternions, Computational Methods and Function Theory, 19, 65-76.

[5] Cerda, G. (2018). Bicomplex Third-Order Jacobsthal quaternions, arXiv: 1809.06979 [math.AC].

[6] Dresden, G.P. \& Du, Z. (2014). A Simplified Binet Formula for $k$-Generalized Fibonacci Numbers, J. Integer Seq., 17, art. 14.4.7, 1-9.

[7] Luna-Elizarrarás, M.E., Shapiro, M., Struppa, D.C., \& Vajiac, A. (2012). Bicomplex Numbers and their Elementary Functions, CUBO A Mathematical Journal, 14 (2), 61-80.

[8] Halıc1, S., \& Karataş, A. (2018). Bicomplex Lucas and Horadam Numbers, arXiv: 1806.05038v2 [math.RA]. 
[9] Halici, S. (2019). On Bicomplex Fibonacci Numbers and Their Generalization. In: Flaut C., Hošková-Mayerová Š., Flaut D. (eds) Models and Theories in Social Systems. Studies in Systems, Decision and Control, Vol 179. Springer, 509-524.

[10] Hathiwala, G.S., \& Shah, D.V. (2017). Binet-Type Formula For The Sequence of Tetranacci Numbers by Alternate Methods, Mathematical Journal of Interdisciplinary Sciences, 6 (1), $37-48$.

[11] Howard, F.T., \& Saidak, F. (2010). Zhou's Theory of Constructing Identities, Congress Numer., 200, 225-237.

[12] Kızılateş, C., Catarino, P., \& Tuğlu, N. (2019). On the Bicomplex Generalized Tribonacci Quaternions. Mathematics, 7, 80.

[13] Melham, R. S. (1999). Some Analogs of the Identity $F_{n}^{2}+F_{n+1}^{2}=F_{2 n+1}^{2}$, Fibonacci Quarterly, 37 (4), 305-311.

[14] Natividad, L. R. (2013). On Solving Fibonacci-Like Sequences of Fourth, Fifth and Sixth Order, International Journal of Mathematics and Computing, 3 (2), 38-40.

[15] Nurkan, S.K., \& Güven İ,A. (2015). A Note on Bicomplex Fibonacci and Lucas Numbers, arXiv: 1508.03972 [math.NT].

[16] Nurkan, S.K., \& Güven İ,A. (2018). A Note on Bicomplex Fibonacci and Lucas Numbers, International Journal of Pure and Applied Mathematics, 120 (3), 365-377.

[17] Rochon, D., \& Shapiro, M. (2004). On Algebraic Properties of Bicomplex and Hyperbolic Numbers, Anal. Univ. Oradea Fascicola. Matematica, 11, 1-28.

[18] Singh, B., Bhadouria, P., Sikhwal, O., \& Sisodiya, K. (2014). A Formula for Tetranacci-Like Sequence, Gen. Math. Notes, 20 (2), 136-141.

[19] Soykan, Y. (2019). Gaussian Generalized Tetranacci Numbers, Journal of Advances in Mathematics and Computer Science, 31 (3), 1-21.

[20] Soykan, Y. (2019). Bicomplex Tetranacci and Tetranacci-Lucas Quaternions, Communications in Mathematics and Applications, 11 (1), 95-112.

[21] Waddill, M.E. (1967). Another Generalized Fibonacci Sequence, Fibonacci Quarterly, 5 (3), 209-227.

[22] Waddill, M.E. (1992). The Tetranacci Sequence and Generalizations, The Fibonacci Quarterly, 30 (1), 9-20.

[23] Zaveri, M.N. \& Patel, J.K. (2016). Binet's Formula for the Tetranacci Sequence, International Journal of Science and Research, 5 (12), 1911-1914. 